\title{
Ethics of Care and Urban Aboriginal and Torres Strait Islander Breastfeeding beyond Three Months
}

\author{
Wendy Foley ${ }^{1 *}$ and Lisa Schubert ${ }^{2}$ \\ ${ }^{1}$ Southern Queensland Centre of Excellence in Aboriginal and Torres Strait Islander Primary Health Care and School of Public Health, The University of \\ Queensland, Australia \\ ${ }^{2}$ School of Public Health, The University of Queensland, Australia
}

Received: October 26, 2015; Accepted: January 07, 2016; Published: January 08, 2016

*Corresponding author: Wendy Foley, Southern Queensland Centre of Excellence in Aboriginal and Torres Strait Islander Primary Health Care and School of Public Health, The University of Queensland, Australia. Tel: +61-7-31817623; E-mail: wendy.foley@health.qld.gov.au

\begin{abstract}
Background: Although early cessation of breastfeeding is common in Australia, as in other industrialised nations, higher proportions of Indigenous mothers cease breastfeeding in the first three months in comparison with other Australian mothers.

Methods: Twenty urban Aboriginal and Torres Strait Islander mothers of infants aged 3 to 12 months participated in in-depth, semistructured interviews which explored the mothers' experiences of infant feeding, and the advice and support they received from health professionals. Data were analysed thematically, using an ethics of care lens.

Results: Three themes arising from the data are presented here: the impact of care on breastfeeding trajectories, relational support, and mother-centred care. Professional care was found to have a strong influence on breastfeeding initiation and duration. Caring relationships with health professionals which prioritise continuity of care and attention to mothers' and infants' specific needs build mothers' trust in their health carers and their own capacities to breastfeed and in turn facilitate positive breastfeeding outcomes. Mother-centred care in hospital, during clinic follow-up, home visits or phone calls facilitates timely identification of mothers' feeding doubts or problems and initiation of solutions to breastfeeding problems. Empathic mother-centred infant feeding care is especially important for mothers with limited support at home.
\end{abstract}

Conclusion: In this research we saw that when health professionals working with urban Aboriginal and Torres Strait Islander mothers demonstrate an ethic of care, which is characterised by culturally safe, mother-centred, relational support, they can positively influence breastfeeding initiation and duration beyond three months. To support urban Indigenous mothers to breastfeed, policy and services need to embrace relational, mother-centred care.

Keywords: Breastfeeding; Breastfeeding Support; Aboriginal and Torres Strait Islander people; Indigenous; Australian; ethic of care; urban

\section{Introduction}

Breastfeeding and human milk provides short- and long-term health benefits for both mother and child. Australian governments recommend exclusive breastfeeding to around six months with the introduction of solid foods then, and continued breastfeeding until about 12 months - and beyond, if both mother and infant wish [1].

In Australia, breastfeeding initiation rates of Aboriginal and Torres Strait Islander infants are close to that of their nonIndigenous counterparts. Although the majority of Australian women do not achieve the recommended breastfeeding duration, higher proportions of Aboriginal and Torres Strait Islander mothers cease breastfeeding during the first few months [2] (Table 1). Moreover, urban Indigenous breastfeeding duration is generally shorter than in remote communities [3,4].

Early breastfeeding cessation is a common phenomenon in industrialised nations [5,6]. The urban Aboriginal and Torres Strait Islander population served by Inala Indigenous Health Service (IIHS), a government health centre serving Aboriginal and Torres Strait Islander people in south-west Brisbane, is no exception. Although breastfeeding prevalence has apparently increased over the last decade, on average, breastfeeding duration in this population remains shorter than in Australia overall (Table 2).

The Aboriginal and Torres Strait Islander breastfeeding initiation rate recorded by IIHS midwives in the year from April 2011 is similar to that previously observed in Indigenous infants in Perth and Melbourne,[7-9] but higher than the 64\% breastfeeding initiation recorded among infants of Aboriginal mothers in NSW hospitals in 2007 [10].

Infant feeding is influenced by multiple individual, family and social factors [11]. Enjoyment of breastfeeding, good support at home, having time to invest in breastfeeding, and having a stable home and a healthy baby, coupled with determination of

Table 1: Exclusive Breastfeeding Prevalence (\%) by Month, Australia, 2010.

\begin{tabular}{|c|c|c|c|c|c|}
\hline & Initiation & $\begin{array}{c}<\mathbf{1} \\
\text { month }\end{array}$ & $\begin{array}{c}<\mathbf{2} \\
\text { months }\end{array}$ & $\begin{array}{c}<\mathbf{3} \\
\text { months }\end{array}$ & $\begin{array}{c}<\mathbf{4} \\
\text { months }\end{array}$ \\
\hline Indigenous & 87 & 59 & 46 & 33 & 19 \\
\hline Non-Indigenous & 90 & 61 & 56 & 48 & 40 \\
\hline \multicolumn{5}{|r|}{ Notes: Source 2010 National Breastfeeding Survey (AIHW 2011) } \\
\hline
\end{tabular}


Table 2: Breastfeeding Prevalence IIHS 1998, 2011 and Australia 2010.

\begin{tabular}{|l|c|c|}
\hline & $\begin{array}{c}\text { Breastfeeding } \\
\text { initiation } \\
\text { (\%) }\end{array}$ & $\begin{array}{c}\text { Any } \\
\text { breastfeeding } \\
\text { after 3 months } \\
\text { (\%) }\end{array}$ \\
\hline Inala 1998* $(n=78)$ & 59 & 35 \\
\hline Inala 2011** $(n=66)$ & 85 & 47 \\
\hline $\begin{array}{l}\text { Australia } 2010^{* * *}(\mathrm{n}= \\
\text { 28,759) }\end{array}$ & 90 & 70 \\
\hline
\end{tabular}

Notes: Data sources: *Hayman, Kanhutu et al. 2000; **IIHS Nurse records, 2011-12; ${ }^{* * *}$ AIHW 2011

the mothers, contribute to sustaining breastfeeding. In Australia, influences on breastfeeding cessation during the first 3 to 6 months are usually reported from the perspective of problems experienced by mother or baby, including anxiety over milk insufficiency, unsettled baby, attachment problems, low maternal breastfeeding satisfaction, poor maternal health and maternal discomfort and inconvenience [12-15]. Some studies have also identified impacts of health system issues on breastfeeding, such as in-hospital midwifery practices and staffing levels $[16,17]$.

Associations between formula feeding and poor health outcomes of mothers and infants throughout their lifespan [18-21], together with the disproportionate burden of disease suffered by Aboriginal and Torres Strait Islander people [22], suggest that an early start to good health through breastfeeding would contribute towards improving long term health outcomes in this population. Appropriate support to increase breastfeeding duration is therefore advocated $[9,15,23,24]$. The aim of this study was to learn from mothers' accounts of infant feeding support and their experiences of initiating, maintaining and ceasing breastfeeding, with a view to informing future infant feeding support in this population.

\section{Methods}

We used qualitative study methods which are described elsewhere [25]. In brief, twenty Aboriginal and Torres Strait Islander mothers of infants aged 3 to12 months were recruited from an Indigenous health service in a disadvantaged urban setting in Brisbane, Queensland. The mothers participated in in-depth, semi-structured interviews (25 to 75 minutes) which explored the mothers' experiences of infant feeding, and the advice and support they received. Interviews were digitally recorded, with consent, and transcribed verbatim. After transcription the first author de-identified the data and checked the transcriptions against the original recordings for accuracy. Codes replaced mothers' names: M1 and M20 being the first and final mothers interviewed, respectively. Transcripts were analyzed thematically using NVivo 9 software [26]. Codes were created inductively by the first author and refined as further data were collected and analyzed. To improve the reliability of the analysis, coding was checked by the second author and further refined on discussion.

For analytical purposes, breastfeeding support is considered in this paper through the lens of an ethics of care, a moral theory which has evolved from the feminist realisation of the importance of caring labour, something generally undervalued in our society [27]. Interpretations of an ethics of care vary [27,28-37]. In this paper we understand care as a value and a practice which can be transformative, rather than merely repetitious [27]. We acknowledge that care takes place within relationships where trust is established through caring practices [27]. This requires that carers be open to those in their care and communicate effectively [35]. Knowing is conceived of as a social process where a recipient of care is listened to in order for the carer to gain knowledge about their specific circumstances, in contrast with scientific traditions of instrumental reasoning where knowledge is understood to facilitate control [38]. An example of this in the context of infant feeding would be providing mothers with written guidelines for 'optimal' infant feeding and expecting compliance to automatically follow.

Caring relationships are frequently unequal and carers can use their power to either control or to empower others. Care ethics encourages analysing the structures and patterns of caring relationships [39]. Practice with an ethics of care requires competence of the carer, attentiveness to and responsiveness of the carer, together with negotiation for solutions to problems $[27,36]$. Such care fosters feelings of control and wellbeing for carers and has the potential to facilitate breastfeeding support.

The Inala Community Jury for Aboriginal and Torres Strait Islander Health Research gave community support to this research before the research began. Ethics approval was granted by Queensland Health's Metro South Human Research Ethics Committee.

\section{Results}

We recruited 20 mothers of infants 3-12 months from an urban Indigenous health service to obtain a varied sample (Table 3).

All mothers initiated breastfeeding, however, by one month, six (30\%) had ceased breastfeeding and from 4 months only 11

\begin{tabular}{|l|c|}
\hline \multicolumn{2}{|l|}{ Table 3: Demographic Characteristics of Study Participants. } \\
\hline Characteristic & Number \\
\hline Mother's age group & 11 \\
\hline $16-19$ & 6 \\
\hline $20-29$ & \\
\hline $30-39$ & 7 \\
\hline Children in family & 6 \\
\hline 1 & 7 \\
\hline $2-3$ & \\
\hline $4-7$ & $10^{*}$ \\
\hline Infant age (months) & 11 \\
\hline $3-6$ & \\
\hline $7-12$ & 8 \\
\hline Infant sex & $13^{*}$ \\
\hline Female & \\
\hline Male & \\
\hline Notes: * Includes twins & \\
\hline
\end{tabular}


(55\%) were feeding their infants any breast milk. We explore below the health service factors which facilitated breastfeeding beyond three months and highlight the experiences of mothers whose breastfeeding duration or intensity for their current infant exceeded their expectations prior to delivery or their experiences feeding previous infants. Three themes arising from the data are considered here: the impact of care on breastfeeding trajectories, relational support and mother-centred care.

\section{The impact of care on breastfeeding trajectories}

Half of the mothers $(n=10)$ breastfed exclusively or predominately beyond 3 months. With one exception, who had confidently breastfed three previous children and managed breastfeeding independently It's just much easier and cheaper to breastfeed. I don't want to spend money on formula and I don't want to get up in the middle of the night. I'd rather sleep. (M3, 33 year old, multipara), these mothers each described receiving tailored, mother-centred care.

Care factors reported by the mothers which facilitated their breastfeeding beyond three months included consistent proactive health sector support, home visits and/or phone calls, encouragement and praise, advocacy with other health staff when needed and tailored breastfeeding advice to meet particular needs. M17, for example, received tailored care which helped her breastfeed her first baby through a difficult initial stage. She was exclusively breastfeeding on her return to work when her baby was 3.5 months:

The midwife that was looking after me ... suggested putting him [in the football hold] on the side, and that was a lot easier for feeding him. ... I was really struggling trying to ... hold him ... and it was very painful as well ... But the football [hold] was the best one that I found. (M17, 29 years old, primipara)

On the other hand, mothers who said that before the birth they had intended to breastfeed, but ceased early, either did not seek help, or received no tailored care when they did. M5, for example, sought support for mastitis but ceased breastfeeding her second infant at about 3 weeks when the nursing support did not help to resolve the problem:

They all said 'Ah - she's on properly,' and I hired a breast pump, but that didn't work.I'm a bit disappointed. (M5, 25 years old, multipara)

Three of the mothers who predominantly breastfed beyond 3 months had not intended to breastfeed. Continuity of care, and caring support encouraged them to initiate and continue breastfeeding:

[child health nurse] ... was supportive through the pregnancy and then after, cause - like he had the heart murmur and stuff and breathing problems - she was like, 'breastfeeding's good for bub, keep it like that - make sure you breastfeed for two years' ... She's like, really supportive. (M19, 26 years old, multipara)

Of the multiparous mothers who breastfed beyond 3 months $(n=8)$, only three reported satisfaction with their previous breastfeeding durations. Four of these 'experienced mothers' increased their breastfeeding intensity and duration with their youngest infant. Caring support offered by health professionals helped them to sustain breastfeeding longer than they had managed with their previous infants:

I [have been breastfeeding twins for 6 months]. Wow ... And I'm still a bit annoyed at myself going, 'Why couldn't I do it with the other ones?' But I did have different support health professionals this time because I didn't really have good support from a health professional around breastfeeding with the other three. So this time it was really ... good. I always felt ... like I was being really promoted to [breastfeed]. (M2, 32 years old, multipara)

Three of the seven primipara exclusively breastfed beyond 3 months. Continuity of breastfeeding care, including home visits, was particularly helpful for them. For example, M10, a 19 year old primipara with no strong intentions to breastfeed recounted:

When I was at home, [clinic midwife] and [lactation consultant made home visits] ... they encouraged me ... And then it started getting a little bit better, but it was still a bit hard. Now he feeds pretty all right.

In contrast, the four who ceased breastfeeding by two months received limited support at pivotal points including postpartum complications, episodes of mastitis /pain, unsettled baby, or family stress and return to work. Some of these mothers sought breastfeeding support, but found it ineffective. Others were not assertive in seeking breastfeeding support or had alternative support available which provided an immediate resolution to feeding problems, but ended breastfeeding. The lack of proactive health sector breastfeeding support for these mothers, and limited attention to educating mothers' support networks allows for support that replaces breastfeeding rather than engage with breastfeeding problem solving.

When mothers internalised the 'breastfeeding rules', yet experienced inadequate support, there were negative breastfeeding outcomes, such as that experienced by M8, a 30 year old primipara who ceased breastfeeding at two months after repeatedly seeking support:

[Nurses] said, 'Keep on persisting,' so I went back there about three more times and basically [was] told to keep on persisting, but I knew she was hungry ... I really wanted to breastfeed but ... [was] frustrated that I couldn't do the job that I was told I had to do.

In this section we have focused on how professional care that is tailored to the needs of mother and infant can positively influence breastfeeding trajectories. In the following sections we will explore approaches to that care.

\section{Relational support}

As described above, M2 was proud of being able to breastfeed twins much longer than her previous singleton infants. A relationship with the supporting health professional, where trust was developed, facilitated this:

I'd sort of look for [child health nurse]. Sometimes somebody else would be there, but I'd go and look for [her] ... I didn't know [her] before I had the kids or anything ... but I just found when I was 
talking to her about breastfeeding ... [she would say] 'you're doing great, it's fantastic'. You know, it was just so positive, whereas [care with previous infants was less positive] ... So I'd go and look for her and sometimes I'd almost look forward to having a weigh in so I could just de-brief. (M2, 32 year old, multipara)

Care where no relationship was established was less positive. M17, a 29 year old primipara, for example, expressed shock and disappointment that she received postnatal home visits from three unfamiliar hospital midwives although she had expected visits from the midwife who cared for her in hospital. Her vulnerable emotional state at this time made it difficult to relate to unfamiliar midwives. Although M17 did continue breastfeeding, interactions with unfamiliar health care professionals, without relationship establishment, discouraged less resilient mothers from seeking further help. M11, initiated breastfeeding her fourth child only on return home from hospital- so she could have a rest in hospital. She was readmitted soon after with a primary postpartum haemorrhage. During this hospitalisation she received no lactation care:

I was getting rushed back because I had a big bleed as well - all my milk drained out ... the second time [in hospital] I didn't mention anything [about breastfeeding], so they didn't ask.

(M11, 27 year old, multipara)

She stopped breastfeeding. M11 had very stressful family circumstances and she and her infant could have benefited from breastfeeding. Although she did not seek breastfeeding support, relational care was lacking and carers made assumptions about her feeding intentions. No hospital carers initiated discussion with her about her family circumstances or infant feeding, which would have been a focus of care provision with an ethic of care.

The contrasting experiences of two 19-year-old primipara also demonstrate how the manner of relating with mothers in hospital can influence their breastfeeding trajectories. M7 expected to breastfeed, but following birth complications struggled to breastfeed for one month. Her experience of postbirth breastfeeding 'care' dissuaded her from seeking further professional support:

They wanted me to [see a lactation consultant], but I didn't want to. Because [nurses] kept pushing the baby and making her cry, and grabbing my breast and that, and I was like, 'You're making me feel really uncomfortable. Go away.' And I thought that that's what the lactation consultant would do, so I was like, 'No!' (M7, 19 year old primipara)

The power imbalance between health professionals and a teenage Indigenous mother is evident. M7 actively resisted the power exerted by nurses over her and her baby by declining further lactation support, despite her initial strong desire to breastfeed. M10, however, who had no preferences about feeding her baby, experienced a more gentle approach from nurses who acknowledged their relationship with her. This encouraged her to accept the proffered care. With ongoing support she breastfed exclusively beyond 3 months:

And [hospital nurses] would express the milk for me and feed it to him. ... They would just do it with their hands. Oh, they would ask me first. They would say, 'Is it all right if we just do it with our hands?' and they did. ... I didn't care really. I was tired. But yeah, he needed to be fed, so whatever got the milk, really. (M10, 19 year old primipara)

Relationship with health professionals advising about breastfeeding was important to most mothers. In contrast to the care she received from familiar nurses at her local clinic, M4 indicated that she would not have called on unfamiliar breastfeeding support networks:

I know there's a lot of [phone] lines for breastfeeding. There's an association isn't there? But yeah, I was just probably too frightened to ring up. (M4, 34 year old, multipara)

Caring weaves people together in relationships [33], and this study found that respectful and responsive caring relationships facilitated positive breastfeeding outcomes.

\section{Mother-centred care}

Attention to individual mothers' specific needs also influenced breastfeeding trajectories. M10, who we saw above, provides an example of this. With little commitment to and trouble initiating breastfeeding, her clinic's lactation consultant and Aboriginal midwife visited regularly, working with her specific needs. She received help with attachment and checking if the baby was feeding well, however the care she received went beyond this:

[Clinic nurses] would come here almost every week, I think. They would stay for ages when they came over here. ... They were really, really good. They helped a lot. She even put [baby] to sleep a few times for me when Mum wasn't home and I couldn't settle him. She'd put him to sleep then leave. I really liked her. (M10, 19 year old, primipara)

The continuity of care and attention to M10's specific needs built trust, in her health carers and her own capacity to continue breastfeeding. Unfortunately not all mothers in the study received this level of care.

Health care empathy and expertise, together with offering encouragement and reassurance when needed, supported mothers to establish and continue breastfeeding. Responding to mothers' uncertainty also facilitated continued breastfeeding:

In the hospital it was hard. Well, especially if you had a $C$-section, you know, it's not all that easy and it also puts stress on yourself and it doesn't feel like you are independent enough to help him. But a couple of days old I sort of got the hang of it but I was still worried that he was not getting enough milk even though it was full and it was coming out right....[Nurses] were just letting me know, it's okay, he is getting the milk. (M12, 23 year old, primipara, breastfed to 6 months, until returning to work.)

Mothers also reported the importance of praise to them:

I took him to get his 6 months needles and ... just the praise, like 'keep breastfeeding' and 'he looks really healthy'. 'Don't put him on the bottle'. Like - which is good, because I have been considering it and knowing that support thing's there. Like - 'keep doing it' makes 
me - oh well, I shouldn't [wean]. (M19, 26 year old, multipara, breastfeeding Breast feeding at 7 months while introducing solids)

Experienced and new mothers responded similarly to reassurance and encouragement:

I liked like when everyone was real positive when he was not feeding good. ... People would just say, "Oh, he'll get it," and stuff like that. And they would - [midwife] convinced me that everyone has trouble anyway. (M10, 19 year old primipara, breastfeeding exclusively at 3 months)

And just with [family problems], it was just hard. I was neglecting myself... And it made it worse when I wasn't producing enough milk that it made me stressed ... [Clinic nurse] rang me up to see how I was going. ... [She] was a good help. She told me she'd check up on me to see if I was doing all right. (M4, 34 year old, multipara breastfed her 4th child after not breastfeeding previously, beyond giving colostrum)

Mother-centred care in the hospital, during regular clinic or home visits and phone calls facilitated timely identification of feeding doubts or problems and the initiation of appropriate solutions to promote continued breastfeeding. This empathic mother-centred infant feeding care was especially important for mothers, like M4, who had limited support at home.

\section{Discussion}

We saw in this study, as elsewhere [40], that health professionals can make a difference to breastfeeding initiation, intensity and duration through working with an ethic of care. For most mothers in this study, care factors during the early weeks and months had a strong impact on breastfeeding outcomes:positive outcomes in terms of breastfeeding beyond 3 months were observed when health professionals practice mother-center, relational care. This approach stands in contrast with one that emphasises guidelines for optimal breastfeeding, which has previously been found to increase maternal stress and undermine confidence [11]. We observed this in mothers for whom breastfeeding did not work well despite following advice given by health professionals. Praise, reassurance, and timely practical help in dealing with unsettled babies, uncertainty about milk insufficiency and breast pain supported some mothers to continue breastfeeding, even when they encountered feeding challenges. Aboriginal and Torres Strait Islander mothers in this study, like mothers elsewhere [41], appreciate reassurance about their breastfeeding, being listened to and having enough time, understanding, and follow-up from health professionals. Health care provision with an ethic of care supports these needs through attentiveness to the specific needs and concerns of mothers and their infants.

Some mothers in this study discussed how health care providers made assumptions around similarities of infant feeding experiences from baby to baby and that experienced mothers knew what they were doing and therefore were in lesser need of instruction or support. We found, however, that breastfeeding support delivered with an ethic of care can be transformative for multiparous mothers. Some mothers in this study fulfilled ambitions to more fully breastfeed their infants. This was facilitated by health professionals' care where expertise was applied to meeting the infant feeding needs in the specific contexts presented by the mother and infant. Within this approach, listening to the mother and providing guidance and encouragement was prioritised over the provision of technical advice and formal breastfeeding guidelines.

In this study we also heard that some mothers were not consistently supported to breastfeed. Mothers did not all experience the same level of care, or persevere to the same extent to seek help for breastfeeding problems, particularly if initial care failed to resolve feeding problems. In these cases formula feeding presented itself as a 'solution' to circumstances when breastfeeding became an inconvenient burden.

Our findings that breastfeeding experiences during the first month after birth have a great impact on the duration of breastfeeding concur with others [42]. A recent Queensland study, however, concluded that health professional contact or home visits in the first ten days post-discharge had no significant impact on breastfeeding at three months [43]. The authors acknowledged that there may be other benefits of health professional contact which they did not assess. Our study adds to this by examining the nature of health professional contact through the lens of an ethic of care. We conclude that it is the nature of the health professional contact, rather than the contact per se, which influences the impact of this interaction. When care is relational and mother-centred it can positively impact on breastfeeding trajectories, as others have also found $[16,44]$.

\section{Implications}

Woman-centred maternity care, defined as 'care that is responsive to women's needs and preferences', is a central principle in the Australian National Maternity Services Plan [45]. Our study confirms that pro-active mother-centred care during the first days and weeks after birth, and at other critical antenatal and postnatal points, is crucial for breastfeeding support for urban Aboriginal and Torres Strait Islander mothers.

If appropriate health sector care is not obtained when it is sought, mothers understandably turn to family and social support, which is not always pro-breastfeeding. The problem-solving skills that these trusted allies share have not necessarily been developed in a strong breastfeeding culture and social factors are pivotal in upholding or abandoning breastfeeding as a normative practice [46]. Health services could recognise the strong role that family and peers play and address this by including these groups in education strategies. Improved management by primary care health professionals of issues which precipitate cessation of breastfeeding, such as unsettled or crying babies, [47], concerns about milk supply, discomfort with feeding and tongue tie [48] could also improve the delivery of infant feeding care so that solutions to problems are informed by good evidence.

This research underscores the importance of staffing capacity to be responsive to the particular circumstances of urban Aboriginal and Torres Strait Islander mothers and 
infants so that consistent, high-quality breastfeeding support is offered. Continuity of care, where relationships of trust are built throughout pregnancy and the early months after birth, is particularly important for achieving culturally competent care for Aboriginal and Torres Strait Islander mothers [45,49]. Implementation in health services of models of care that support continuity of care and building relationships with mothers would contribute to supporting urban Indigenous mothers in breastfeeding initiation and establishment. Midwifery staffing levels, however, often serve to limit the formation of meaningful relationships with mothers [50,51]. Moreover, since post-birth care in Australia is fragmented [47], bridging the gaps between hospitals and local clinics is vital [48] and could help to ensure continuity of care for improved breastfeeding outcomes.

The National Maternity Services Plan [45] acknowledges that Aboriginal and Torres Strait Islander people experience considerable health inequalities and social disadvantage, which can lead to less favourable outcomes for these mothers and babies. Its 5 year vision articulated the need for culturally competent women-centred care to close the gap between health outcomes from Indigenous people and their non-Indigenous counterparts. We endorse the importance of this vision, however, at the end of the period encompassed by this plan; this study indicates that more work is needed to fulfil this vision in urban Indigenous settings.

Professional caring relationships are often unequal, and especially so when the recipients of care come from disadvantaged groups. How this additional inequality impacts on care needs to be further explored and addressed through working out the requirements for health services to effectively operationalize mother-centred care for urban Indigenous mothers.

Some mothers in this study indicated they did not feel empowered to seek the breastfeeding support they needed. For disadvantaged urban Indigenous mothers therefore, breastfeeding support needs to be implemented in ways which facilitate equity of outcomes. Health professionals need to be able to work in culturally safe ways in this population, prioritising care which facilitates building relationships, enabling responsiveness to the mothers and sensitive negotiation of strategies to address breastfeeding challenges which would work in their specific circumstances. The provision of relevant and consistent information and physical care, home visits and/or phone calls when needed and facilitating opportunities for pro-breastfeeding support into ante- and postnatal peer network groups are important complements to this support. Given the relative socioeconomic disadvantage experienced by many urban Indigenous people [46,52], private sector services are beyond the economic means of most. Therefore the investment of publically-funded health sector resources to strengthen relational, mother-centred support is particularly important to empower urban Aboriginal and Torres Strait Islander mothers and their networks to effectively address breastfeeding challenges.

\section{Strengths and limitations}

This paper adds to the scant research on urban Indigenous infant feeding. While it presents findings from a small sample of mothers in one urban Indigenous health service, strength of this research is that it employed in-depth interviews, giving voice to urban Indigenous women's stories about their infant feeding experiences. A limitation of this study is that mothers recruited to the study had infants ranging in age from 3 to 12 months and the retrospective recall about early feeding issues could have been compromised for those with the older infants. A prospective study in this area, visiting mothers at intervals during the first year could be used to minimise recall issues.

\section{Conclusion}

Supporting mothers to feed their infants is an important element of maternity and postnatal care. However, services can be disjointed and unresponsive, leaving some urban Aboriginal and Torres Strait Islander mothers to cope the best way they can. This study points to the fact that when mothers are empowered by health professionals, who take a caring approach that puts the mothers and their needs at the centre of the care, they can overcome breastfeeding challenges to establish comfortable feeding patterns, and sometimes even breastfeed more than they had expected or achieved with previous infants. Providing such care for all Indigenous mothers should be a priority.

\section{References}

1. Australian Government. (2012). Breastfeeding. Retrieved 11 Feb, 2013, from http://www.health.gov.au/breastfeeding

2. AIHW. (2011). 2010 Australian national infant feeding survey: indicator results. Retrieved 19 January, 2012, from http://www. aihw.gov.au/publication-detail/?id=10737420927\&libID=10737420 $926 \&$ tab $=2$

3. Australian Government. Footprints in Time: The Longitudinal Study of Indigenous Children. Report from Wave 4. Canberra: Australian Government. 2013.

4. Cromie EA, Shepherd CC, Zubrick SR, Oddy WH. Breastfeeding Duration and Residential Isolation amid Aboriginal Children in Western Australia. Nutrients. 2012; 4(12): 2020-34. doi: 10.3390/ nu4122020.

5. Perrine CG, Scanlon KS, Li R, Odom E, Grummer-Strawn LM. BabyFriendly Hospital Practices and Meeting Exclusive Breastfeeding Intention. Pediatrics. 2012; 130 (1): 54-60. doi: 10.1542/peds.20113633.

6. Renfrew MJ, Spiby H, D’Souza L, Wallace LM, Dyson L, McCormick F. Rethinking research in breast-feeding: a critique of the evidence base identified in a systematic review of interventions to promote and support breast-feeding. Public Health Nutr. 2007; 10(7): 726-32.

7. Binns C, Gilchrist D, Gracey M, Zhang M, Scott J, Lee A. Factors associated with the initiation of breast-feeding by Aboriginal mothers in Perth. Public Health Nutr. 2004; 7(7): 857-61.

8. Eades SJ, Read AW, McAullay D, McNamara B, O’Dea K, Stanley FJ. Modern and traditional diets for Noongar infants. J Paediatr Child Health. 2010; 46(7-8): 398-403. doi: 10.1111/j.14401754.2010.01752.x

9. Holmes W, Phillips J, Thorpe L. Initiation rate and duration of breastfeeding in the Melbourne Aboriginal community. Aust N Z J Public Health. 1997; 21(5): 500-3. 
10. Taylor LK, Lim K, Neville SE. Newborn feeding practices at the time of discharge from hospital in NSW in 2007: a descriptive study. N S W Public Health Bull. 2009; 20(11-12): 177-81. doi: 10.1071/NB09024.

11. Hoddinott P, Craig LC, Britten J, McInnes RM. A serial qualitative interview study of infant feeding experiences: idealism meets realism. BMJ Open. 2012; 2(2): e000504. doi: 10.1136/bmjopen-2011-000504.

12. Australian Institute of Health and Welfare. (2011). 2010 Australian national infant feeding survey: indicator results. (Cat. No. PHE 156). Canberra: AIHW Retrieved from http://www.aihw.gov.au/ WorkArea/DownloadAsset.aspx?id=10737420925

13. Colin WB, Scott JA. Breastfeeding: Reasons for starting, reasons for stopping and problems along the way. Breastfeed Rev. 2002; 10(2): 13-9.

14. Cooke M, Sheehan A, Schmied V. A Description of the Relationship between Breastfeeding Experiences, Breastfeeding Satisfaction, and Weaning in the First 3 Months after Birth. J Hum Lact. 2003; 19(2): 145-56.

15. Eades, Sandra J. Breastfeeding among urban Aboriginal women in Western Australia. Aboriginal and Islander Health Worker Journal. 2000; 24(3): 9-14.

16. Gilmour C, Hall H, McIntyre M, Gillies L, Harrison B. Factors associated with early breastfeeding cessation in Frankston, Victoria: a descriptive study. Breastfeed Rev. 2009; 17(2): 13-9.

17. McAllister H, Bradshaw S, Ross-Adjie G. A study of in-hospital midwifery practices that affect breastfeeding outcomes. Breastfeed Rev. 2009; 17(3): 11-5.

18. Chantry CJ, Howard CR, Auinger P. Full breastfeeding duration and associated decrease in respiratory tract infection in US children. Pediatrics. 2006; 117(2): 425-32.

19. Hoddinott P, Tappin D, Wright C. Breast feeding. BMJ. 2008; 336(7649): 881-7. doi: 10.1136/bmj.39521.566296.BE.

20. Schwarz EB, Brown JS, Creasman JM, Stuebe A, McClure CK, Van Den Eeden SK, et al. Lactation and Maternal Risk of Type 2 Diabetes: A Population-based Study. Am J Med. 2010; 123(9): 863.e1-6. doi: 10.1016/j.amjmed.2010.03.016.

21. Scott JA, Ng SY, Cobiac L. The relationship between breastfeeding and weight status in a national sample of Australian children and adolescents. BMC Public Health. 2012; 12: 107. doi: 10.1186/14712458-12-107.

22. MacRae A, Thomson N, Anomie Burns J, Catto M, Gray C, Urquhart B. 2013. Overview of Australian Indigenous health status, 2012. Retrieved 10 January, 2014, from http://www.healthinfonet.ecu.edu. $\mathrm{au} /$ health-facts/overviews

23. Hayman N, Kanhutu J, Bond S, Marks GC. Breast-feeding and weaning practices of an urban community of Indigenous Australians. Asia Pac J Clin Nutr. 2000; 9(3): 232-4.

24. Rogers L. Understanding barriers to breastfeeding in Aboriginal and Torres Strait Islander women. Aboriginal and Islander Health Worker Journal. 2006; 30(1): 8-9, 32.

25. Foley W, Schubert L, Denaro T. Breastfeeding experiences of Aboriginal and Torres Strait Islander mothers in an urban setting in Brisbane. Breastfeed Rev. 2013; 21(3): 53-61.

26. QSR International Pty Ltd. NVivo qualitative data analysis software; Version 9. 2010.

27. Held V. (2006). The ethics of care: personal, political, and global
Retrieved from http://www.oxfordscholarship.com.ezproxy. library.uq.edu.au/view/10.1093/0195180992.001.0001/ acprof-9780195180992

28. Allmark P. Can there be an ethics of care? J Med Ethics. 1995; 21(1): 19-24.

29. Bradshaw A. Yes! There is an ethics of care: an answer for Peter Allmark. J Med Ethics. 1996; 22(1): 8-15.

30. Edwards SD. Three versions of an ethics of care. Nurs Philos. 2009; 10(4): 231-40. doi: 10.1111/j.1466-769X.2009.00415.x.

31.Edwards SD. Is there a distinctive care ethics? Nurs Ethics. 2011; 18(2): 184-91. doi: 10.1177/0969733010389431.

32. Kittay EF. The Ethics of Care, Dependence, and Disability. Ratio Juris. 2011; 24(1): 49-58. doi: 10.1111/j.1467-9337.2010.00473.x.

33. Lachman VD. Applying the Ethics of Care to Your Nursing Practice. Medsurg Nurs. 2012; 21(2): 112-4, 116.

34. Parker RS. Nurses' stories: the search for a relational ethic of care. ANS Adv Nurs Sci. 1990; 13(1): 31-40.

35. Parton N. Rethinking Professional Practice: The contributions of Social Constructionism and the Feminist 'Ethics of Care'. Brit J Soc Work. 2003; 33(1): 1-16. doi: 10.1093/bjsw/33.1.1.

36. Tronto JC. An ethic of care. Generations. 1998; 22(3): 15-20.

37. Vanlaere L, Gastmans C. A personalist approach to care ethics. Nurs Ethics. 2011; 18(2): 161-73. doi: 10.1177/0969733010388924.

38. Rima D Apple. Perfect Motherhood: Science and Childrearing in America. New Brunswick, NJ: Rutgers University Press. 2007. DOI: 10.1111/j.1741-3737.2007.00453.x.

39. ethicsofcare.org. (2006). Interviews: Ruth Groenhout. Retrieved 10 January, 2014, from http://ethicsofcare.org/interviews/ruthgroenhout/

40. Fisher P, Owen J. Empowering interventions in health and social care: recognition through 'ecologies of practice'. Soc Sci Med. 2008; 67(12): 2063-71. doi: 10.1016/j.socscimed.2008.09.035.

41. Bäckström CA, Wahn EI, Ekström AC. Two sides of breastfeeding support: experiences of women and midwives. Int Breastfeed J. 2010; 5: 20. doi: 10.1186/1746-4358-5-20.

42. Baxter J, Cooklin AR, Smith J. Which mothers wean their babies prematurely from full breastfeeding? An Australian cohort study. Acta Paediatr. 2009; 98(8): 1274-7. doi: 10.1111/j.16512227.2009.01335.x.

43. Brodribb WE, Miller YD. The Impact of Community Health Professional Contact Postpartum on Breastfeeding at 3 Months: A Cross-Sectional Retrospective Study. Matern Child Health J. 2014; 18(7): 1591-8. doi: 10.1007/s10995-013-1398-3.

44. Kervin BE, Kemp L, Pulver LJ. Types and timing of breastfeeding support and its impact on mothers' behaviours. J Paediatr Child Health. 2010; 46(3): 85-91. doi: 10.1111/j.1440-1754.2009.01643.x.

45. AHMAC. National Maternity Services Plan. Canberra: Australian Health Ministers' Council, Commonwealth of Australia. 2011.

46. Biddle, Nicholas. Same suburb, different worlds: Comparing Indigenous and non-Indigenous outcomes in city suburbs and large regional towns. People and Place. 2009; 17(2): 56-63.

47. Douglas PS, Mares RE, Hill PS. Interdisciplinary perspectives on the management of the unsettled baby: key strategies for improved 
outcomes. Aust J Prim Health. 2012; 18(4): 332-8.

48. Edmunds J, Miles SC, Fulbrook P. Tongue-tie and breastfeeding: a review of the literature. Breastfeed Rev. 2011; 19(1): 19-26.

49. Kruske S. Culturally Competent Care for Aboriginal and Torres Strait Women Report 2012. Canberra: Maternity Services InterJurisdictional Committee for the Australian Health Ministers' Advisory Council. 2013.

50. Hailes JF, Wellard SJ. Support for breastfeeding in the first postpartum month: perceptions of breastfeeding women. Breastfeed Rev. 2000;
8(3): 5-9.

51. Hauck YL, Bayes SJ, Robertson JM. Addressing the workplace needs of Western Australian midwives: a Delphi study. Aust Health Rev. 2012; 36(2): 176-83. doi: 10.1071/AH11026.

52. Nicholas Biddle. CAEPR Indigenous Population Project: 2011 Census Papers - Socioeconomic outcomes. Canberra: Australian National University, Centre for Aboriginal Economic Policy Research, Research School of Social Sciences. 2013. 